Domínguez Alonso, J., Álvarez Roales, E. y Vázquez Varela, E. (2017). Dimensiones predictivas del constructo violencia escolar en la educación secundaria obligatoria. Revista de Investigación Educativa, 35(2), 337-351 DOI: http://dx.doi.org/10.6018/rie.35.2.259471

\title{
Dimensiones predictivas del constructo violencia escolar en la educación secundaria obligatoria
}

\section{Predictors of violence in compulsory secondary education}

\author{
José Domínguez Alonso*, Enrique Álvarez Roales**, Elia Vázquez Varela*** \\ *Departamento de Análisis e Intervención Educativa. Universidad de Vigo (España) \\ ** Departamento de Análisis e Intervención Educativa. Universidad de Vigo (España) \\ *** Departamento de Análisis e Intervención Educativa. Universidad de Vigo (España)
}

\begin{abstract}
Resumen
Introducción. Las causas que han originado la violencia en los centros educativos son muy diversas y a menudo interrelacionadas. El presente estudio analiza la capacidad de predicción de las diferentes dimensiones que conforman el constructo violencia escolar. Método. Para identificar las dimensiones predictivas de la violencia escolar se realizó un análisis de regresión lineal múltiple. Participaron 4.943 estudiantes de Educación Secundaria Obligatoria (51.2\% chicas y 48.8\% chicos) de entre 13 y 16 años (Media = 14.04; DT =1.38) de la Comunidad Autónoma de Galicia (España), los cuales respondieron al cuestionario de violencia escolar CUVE3-ESO. Resultados. Los resultados sugieren que la violencia escolar se puede predecir en mayor medida por las dimensiones: violencia del alumnado hacia el alumnado (explica un $27.28 \%$ de la varianza) y violencia del alumnado hacia el profesorado y amenazas entre estudiantes (explica un $21.06 \%$ de la varianza). Finalmente, es significativa pero escasa la aportación de las cinco dimensiones restantes: violencia del profesorado hacia el alumnado (3.92\% de la varianza), disrupción en el aula $(3.68 \%$ de la varianza), violencia física del alumnado $(2.84 \%$ de la varianza), exclusión social (1.52\% de la varianza) y violencia física indirecta (1.42\% de la varianza). Discusión. Los análisis de regresión revelaron que los escolares implicados en conductas violentas hacia sus compañeros o profesorado presentan una mayor predisposición de ejercer violencia escolar. Estos novedosos resultados muestran la importancia de incluir la violencia del alumnado en los programas de prevención de la conflictividad en los centros educativos.
\end{abstract}

Correspondencia: José Domínguez Alonso, Universidad de Vigo, España. jdalonso@uvigo.es. 
Palabras clave: Violencia entre escolares; educación secundaria; relaciones intergrupales; análisis de regresión.

\begin{abstract}
Introduction. The causes that lead to violence in schools are very diverse and often interrelated. The current study analyzes the predictive ability of different dimensions which are found within the school violence framework. Method. A multiple linear regression analysis was performed in order to identify the predictors of school violence. 4,943 Compulsory Secondary Education students participated in the study (51.2\% girls and 48.8\% boys) aged between 13 and 16 years (Mean $=14.04, S D=1.38$ ), from the Autonomous Community of Galicia (Spain), who responded to the questionnaire on school violence, CUVE3 - CSE. Results. The results suggest that school violence can be predicted to a greater extent by applying the following dimensions: peer violence (accounting for $27.28 \%$ of the variance) and students' violence toward teachers and threats among students (accounting for $21.06 \%$ of the variance). Finally, the contribution of the remaining five predictors is significant, but minimal: teacher violence toward students (3.92\% of the variance), classroom disruption (3.68\% of the variance), students' physical violence (2.84\% of the variance), social exclusion (1.52\% of the variance) and indirect physical violence (1.42\% of the variance). Discussion. Regression analyses revealed that students involved in violent behavior toward their peers or teachers are more likely to utilize school violence. These new results show the importance of including students' violence in conflict prevention programs in schools.

Keywords: Peer Violence; Secondary Education; Intergroup Relationships; Regression Analysis.
\end{abstract}

\title{
Introducción
}

Los recientes cambios educativos y normativos han tenido un impacto importante sobre la violencia en los centros de enseñanza, los cuales no sólo han de mejorar las relaciones entre sus estudiantes, también se les exige la capacidad de aportar soluciones para gestionar situaciones de conflictividad en estos espacios formativos. Mientras que en el pasado las viejas formas de regular la conflictividad y violencia han sido puestas en entredicho, tampoco las nuevas iniciativas parecen alcanzar la eficacia sospechada. De ahí la relevancia de incorporar nuevas investigaciones como eje de análisis en la prevención (Cooley-Strickland, Griffin \& Furr-Holden, 2011; Gracia \& Herrero, 2006) e intervención (Abramovay, 2005; Arce, Seijo, Fariña, Mohamed-Mohand, 2010; Gázquez, Pérez-Fuentes, Carrión \& Santiuste, 2010) de la violencia escolar.

Así pues, la violencia escolar, un constructo tan relevante como multifactorial que se manifiesta fundamentalmente como un proceso de interacción más que como un fenómeno individual, ha registrado un claro avance en la última década como una perspectiva transversal en los centros educativos (Palmero, Guerrero, Gómez, Carpi \& Gorayeb, 2010). Asimismo, aunque los índices de conflictividad en los centros educativos tienden a ser bajos (Avilés, 2009; Cerezo, 2009; Perren, Dooley, Shaw \& Cross, 2010; Sanchéz-Lacasa \& Cerezo, 2010), han alcanzado gran notoriedad en la última década (Avilés \& Monjas, 2005; Defensor del Pueblo-UNICEF, 2007; Guil \& Mestre, 2004; Ortega \& Mora-Merchán, 2008; Torrego \& Moreno, 2003). 
Por consiguiente, el estudio de la violencia escolar, actualmente en una etapa de expansión mediática, se ha convertido en un tema de potencial interés (Shapiro, 2006), llegando a ser considerada uno de los principales problemas de salud pública y educación en todo el mundo (Cajigas de Segredo et al., 2006). En la mayoría de investigaciones se pone de manifiesto que la violencia escolar incide más en la etapa de educación secundaria obligatoria (Benitez \& Justicia, 2006; Calvete, Orue, Estévez, Villardón \& Padilla, 2010; Díaz-Aguado, 2005; Estévez, 2006), presentando graves consecuencias en el desarrollo y ajuste psicosocial de los adolescentes (Cava, Buelga, Musitu \& Murgui, 2010; Del Moral, Suárez \& Musitu, 2012; Ortega, 2010).

En términos generales, las conductas violentas surgen de un ejercicio de dominio a través del uso de la fuerza y se dan en contextos de desigualdad y desequilibrio de poder, que puede acrecentarse en los centros educativos, en tanto a su constitución como sistema social de mayor amplitud. No obstante, debido a lo versátil y ubicuo del término violencia escolar, es difícil delimitarlo con exactitud (Torrego, 2007). En consecuencia, el constructo violencia escolar, tan relevante como controvertido, ha sido conceptualizado de distintas maneras por diversos autores haciendo referencia a "cualquier relación, proceso o condición por la cual un individuo o un grupo social viola la integridad física, psicológica o social de otra persona" (Rivera, 2011, p. 20) y que altera en mayor o menor medida el ámbito escolar (Martínez-Otero, 2005; MartínSeoane, 2008).

Para este estudio, vamos a centrarnos en el concepto de violencia escolar, conocida como aquella conducta u omisión intencionada con la que se causa un daño o perjuicio (Álvarez-García et al., 2008) y agrupa a múltiples formas de manifestarse en los centros educativos. De hecho, siguiendo a Álvarez-García, Núñez, y Dobarro (2013), se hará referencia a: violencia física (existe contacto material para producir daño), diferenciando la que ejerce contacto directo sobre la víctima (violencia física directa) o la que el contacto es sobre material o pertenencias (violencia física indirecta); violencia verbal (el daño se causa mediante la palabra); exclusión social (actos de discriminación y rechazo); y disrupción en el aula (comportamientos con los que el alumnado dificulta al profesorado impartir clase y al resto de compañeros interesados seguirla con aprovechamiento).

\section{Método}

\section{Objetivos}

La presencia de la violencia escolar y sus posibles manifestaciones en los centros de enseñanza es, sin duda, uno de los grandes desafíos a los que hay que enfrentarse desde las comunidades educativas. Dicho esto, el objetivo del presente trabajo de investigación es identificar las dimensiones predictoras del constructo violencia escolar en los centros de educación secundaria desde la perspectiva del alumnado.

\section{Población y Muestra}


A efectos de nuestro estudio, la población queda definida por el alumnado matriculado en Educación Secundaria Obligatoria (ESO) durante el curso 2013-2014 en la Comunidad Autónoma de Galicia (España). La imposibilidad de que todo el alumnado pudiese participar en la investigación [ $\mathrm{N}=88.529$ (Xunta de Galicia, 2014)], lleva a utilizar un muestreo propositivo sin fijar a priori el tamaño (Creswell, 2009; Neuman, 2009). Finalmente, la muestra quedó formada por un total de 4943 alumnos, de los cuales 2411 son hombres $(48.78 \%$ ) y 2532 mujeres (51.22\%). La media de edad expresada en años es de $14.05 \pm 1.38$. Además, según el curso 1336 estudiantes estaban matriculados en $1^{\circ}$ de ESO (27\%), 1291 en $2^{\circ}$ de ESO (26.1\%), 1262 en $3^{\circ}$ de ESO (25.5\%) y 1054 en $4^{\mathrm{o}}$ de $\mathrm{ESO}(21.3 \%)$

\section{Instrumento}

En primer lugar, se ha elaborado y aplicado un cuestionario complementario ("ad hoc") con el objeto de conocer distintos datos demográficos de los individuos de la muestra.

En segundo lugar, para la realización de este estudio se ha utilizado el Cuestionario de Violencia Escolar, CUVE3-ESO, (Álvarez-García, Núñez \& Dobarro, 2012), que permite analizar la frecuencia de aparición de diferentes tipos de violencia escolar, protagonizada por el alumnado o el profesorado (Ver ANEXO). Se ha llevado a cabo para la muestra obtenida estudios de validez y fiabilidad del cuestionario. En primer lugar, se realizó un Análisis Factorial de Componentes Principales obteniendo valores adecuados para el índice KMO (.961) como en el test de esfericidad de Barlett $\left(\chi_{946}^{2}=96870.64 ; p<.001\right)$. La estructura dimensional resultante quedó conformada por siete factores que conjuntamente explican un $55.48 \%$ de la varianza. A continuación se procedió a la realización del análisis factorial confirmatorio del modelo teórico hipotetizado resultante del Análisis Factorial Exploratorio, presentando unos índices de ajuste correctos $\left[\mathrm{x}^{2} / \mathrm{gl}=12.247\right.$ (condicionado por el tamaño de la muestra); GFI=.93; CFI=.91; RMSEA=.04].

En resumen, el cuestionario compuesto por 44 ítems que adoptan un formato de respuesta tipo Likert de cinco alternativas (nunca, pocas veces, algunas veces, muchas veces, siempre) ha quedado conformado por siete dimensiones: Violencia del Profesorado hacia el Alumnado (VPA: 10 ítems, por ej. "el profesorado tiene manía al alumnado"), Violencia del Alumnado hacia el Profesorado y Amenazas entre Estudiantes (VAPAE: 9 ítems, por ej. "los estudiantes roban cosas al profesorado"), Violencia del Alumnado hacia el Alumnado (VAA: 8 ítems, por ej. "estudiantes amenazan a través de las redes sociales a sus compañeros"), Disrupción en el Aula (DA: 7 ítems, por ej. "el alumnado está continuamente dificultando las explicaciones del profesorado"), Exclusión Social (ES: 4 ítems, por ej. "el alumnado es discriminado por cuestión de nacionalidad"), Violencia Física entre el Alumnado (VFA: 3 ítems, por ej. "el alumnado se pelea dentro del recinto escolar"), y Violencia Física Indirecta (VFI: 3 ítems, por ej. "el alumnado pone motes molestos a compañeros").

Finalmente, la fiabilidad del instrumento fue medida a través del alpha de Cronbanch, obteniendo un valor de .95 para los 44 ítems (VPA: .89; VAPAE: .87; VAA: .86; DA: .82; ES: .72; VFA: .80; VFI: .77), por encima del límite inferior que es aceptado como fiable (Corbetta, 2007). 


\section{Procedimiento de recogida y análisis de datos}

En primer lugar se ha establecido una reunión con los equipos directivos de los centros educativos para solicitar su libre colaboración en el estudio, sin ningún tipo de coacción. El alumnado fue avisado del anonimato de las respuestas y se hizo especial hincapié en la confidencialidad de los resultados. La aplicación fue colectiva por el propio equipo de investigación, con el fin de fomentar la respuesta al cuestionario, sinceridad y correcta contestación. Este estudio lleva aparejado a seres humanos, por lo cual los aspectos éticos son relevantes. Al respecto, los participantes en la misma fueron informados del propósito del trabajo, consentimiento o aprobación, y sobre todo, confidencialidad y anonimato. Asimismo, se han obtenido los debidos permisos para acceder a los centros educativos.

Dado el objetivo principal de este trabajo, el estudio se basa en una investigación de tipo empírico (Ato, López \& Benavente, 2013), empleando un análisis de regresión lineal múltiple por el método stepwise (pasos sucesivos), con el propósito de determinar el valor predictivo de las distintas dimensiones que conforman el constructo de la violencia escolar, cumpliendo los supuestos de linealidad, independencia, normalidad, homocedasticidad y no colinealidad, para garantizar la validez del modelo. Los datos fueron analizados con el paquete informático SPSS 22 (SPSS Inc., Chicago, USA).

\section{Resultados}

Los resultados van a ser presentados en función del objetivo planteado. Han sido excluidas las variables demográficas del análisis de regresión realizado, debido a no constituir ninguna dimensión del constructo violencia escolar en este estudio.

\section{Violencia ejercida por el profesorado hacia el alumnado y viceversa}

En primer lugar (tabla 1), teniendo en cuenta la violencia ejercida por el profesorado sobre el alumnado (VPA), los resultados aportados por el análisis de regresión indican que se han incluido las dimensiones violencia del alumnado hacia el profesorado y amenazas entre estudiantes (VAPAE), violencia física entre el alumnado (VFA), violencia física indirecta (VFI), violencia del alumnado hacia el alumnado (VAA) y disrupción en el aula (DA); quedando descartada la exclusión social (ES). Teniendo en cuenta el orden de entrada de cada dimensión en la ecuación final de regresión (que indica también el orden de su relevancia o poder predictivo), han mostrado cierto poder predictor la VAPAE $(\beta=.21 ; t=12.38 ; p<.01)$, la VFA $(\beta=.14 ; t=8.52 ; p<.01)$, la VFI $(\beta=.13 ; t=8.35 ; p<.01)$, la VAA $(\beta=.11 ; t=5.49 ; p<.01)$ y la DA $(\beta=.10 ; t=5.04 ; p<.01)$. Estas dimensiones, en conjunto, explican el 30\% de la varianza total de la violencia ejercida por el profesorado sobre el alumnado. Asimismo, atendiendo a la cantidad de varianza explicada por cada dimensión, se observa que la primera en entrar en la ecuación (VAPAE) explica el 22\% del total, siendo significativa pero escasa la aportación de las cuatro dimensiones restantes (entre un $4.25 \%$ y un .55\%). El coeficiente de correlación es de .54 , lo que indica una relación considerable y positiva entre las dimensiones. 
Tabla 1

Análisis de regresión lineal (pasos sucesivos), dimensiones criterio: VPA y VAPAE

\begin{tabular}{|c|c|c|c|c|c|c|c|}
\hline $\begin{array}{c}\text { Dimensión } \\
\text { criterio }\end{array}$ & $R$ & $R^{2}$ & $F$ & \multirow{2}{*}{$\begin{array}{l}\text { Dimensiones } \\
\text { Predictoras }\end{array}$} & \multirow[t]{2}{*}{$\beta$} & \multirow[t]{2}{*}{$t$} & \multirow{2}{*}{$\begin{array}{l}\text { Varianza } \\
\text { Explicada }\end{array}$} \\
\hline VPA & .54 & .30 & $412.88(p<.001)$ & & & & \\
\hline & & & & $\begin{array}{l}\text { VAPAE } \\
\text { VFA } \\
\text { VFI } \\
\text { VAA } \\
\text { DA }\end{array}$ & $\begin{array}{l}.21 \\
.14 \\
.13 \\
.11 \\
.10\end{array}$ & $\begin{array}{l}12.38^{* *} \\
8.52^{* *} \\
8.35^{* *} \\
5.49^{* *} \\
5.04^{* *}\end{array}$ & $\begin{array}{c}22 \% \\
4.25 \% \\
2.25 \% \\
.95 \% \\
.55 \%\end{array}$ \\
\hline \multirow[t]{2}{*}{ VAPAE } & .75 & .57 & $1268.81(p<.001)$ & & & & \\
\hline & & & & $\begin{array}{l}\text { VAA } \\
\text { ES } \\
\text { VFA } \\
\text { VPA } \\
\text { VFI }\end{array}$ & $\begin{array}{l}.35 \\
.22 \\
.18 \\
.13 \\
.06\end{array}$ & $\begin{array}{c}25.14^{* *} \\
18.18^{* *} \\
14.77^{* *} \\
11.52^{* *} \\
4.95^{* *}\end{array}$ & $\begin{array}{c}47 \% \\
5.03 \% \\
3.29 \% \\
1.39 \% \\
.29 \%\end{array}$ \\
\hline
\end{tabular}

Nota: VPA: Violencia del Profesorado hacia el Alumnado; VAPAE: Violencia del Alumnado hacia el Profesorado y Amenazas entre Estudiantes; VFA: Violencia Física entre el Alumnado; VFI: Violencia Física Indirecta; DA: Disrupción en el Aula; VAA: Violencia del Alumnado hacia el Alumnado. ES: Exclusión Social; ${ }^{* *}=p<.01$.

En segundo lugar, la violencia del alumnado hacia el profesorado y amenazas entre estudiantes (VAPAE) es predicha también por cinco dimensiones (tabla 1): VAA ( $\beta=.35$; $t=25.14 ; p<.01)$, ES $(\beta=.22 ; t=18.18 ; p<.01)$, VFA $(\beta=.18 ; t=14.77 ; p<.01), \mathrm{VPA}(\beta=.13 ; t=11.52$; $p<.01)$ y VFI ( $\beta=.06 ; t=4.95 ; \beta<.01)$; quedando excluida la disrupción en el aula (DA). En conjunto, las cinco dimensiones explican el $57 \%$ de la varianza total de la VAPAE, aunque al igual que en el caso de la VPA, la violencia del alumnado hacia el alumnado es la dimensión más importante en la predicción de la VAPAE (47\%). Aquí, el coeficiente de correlación es de .75 , lo que indica una relación alta y positiva entre dimensiones.

\section{Violencia entre el alumnado}

En cuanto a la predicción de la violencia entre el alumnado (violencia del alumnado hacia el alumnado y violencia física del alumnado), los resultados obtenidos muestran (tabla 2), una vez más, que en la VAA la dimensión con mayor poder de predicción es la VAPAE $(\beta=.30 ; t=24.46 ; \beta<.01)$, y en un nivel menor están la DA $(\beta=.24 ; t=21.85$; $p<.01)$, la VFI $(\beta=.16 ; t=14.90 ; \beta<.01)$, la ES $(\beta=.14 ; t=13.05 ; p<.01)$, la VFA $(\beta=.14 ; t=11.57$; $p<.01)$ y la VPA $(\beta=.05 ; t=5.17 ; \beta<.01)$. La primera dimensión (VAPAE) y la segunda (DA) explican el $57.48 \%$ de la varianza total de la violencia del alumnado hacia el alumnado (VAA). Señalar que el coeficiente de correlación es .80, apuntando a una relación alta y positiva entre dimensiones. 
Tabla 2

Análisis de regresión lineal (pasos sucesivos), dimensiones criterio: VAA y VFA

\begin{tabular}{|c|c|c|c|c|c|c|c|}
\hline $\begin{array}{c}\text { Dimensión } \\
\text { criterio }\end{array}$ & $R$ & $R^{2}$ & $F$ & \multirow{2}{*}{$\begin{array}{c}\text { Dimensiones } \\
\text { Predictoras }\end{array}$} & \multirow[t]{2}{*}{$\beta$} & \multirow[t]{2}{*}{$t$} & \multirow{2}{*}{$\begin{array}{l}\text { Varianza } \\
\text { Explicada }\end{array}$} \\
\hline \multirow[t]{7}{*}{ VAA } & .80 & .64 & $1439.63(p<.001)$ & & & & \\
\hline & & & & VAPAE & .30 & $24.46^{* *}$ & $46 \%$ \\
\hline & & & & $\mathrm{DA}$ & .24 & $21.85^{* *}$ & $11.48 \%$ \\
\hline & & & & VFI & .16 & $14.90^{* *}$ & $3.18 \%$ \\
\hline & & & & ES & .14 & $13.05^{* *}$ & $1.68 \%$ \\
\hline & & & & VFA & .14 & $11.57^{* *}$ & $1.28 \%$ \\
\hline & & & & VPA & .05 & $5.17^{* *}$ & $.38 \%$ \\
\hline \multirow[t]{7}{*}{ VFA } & .70 & .49 & $776.77(p<.001)$ & & & & \\
\hline & & & & VAA & .19 & $11.57^{* *}$ & $38 \%$ \\
\hline & & & & VAPAE & .22 & $14.79^{* *}$ & $4.80 \%$ \\
\hline & & & & DA & .21 & $15.25^{* *}$ & $3.90 \%$ \\
\hline & & & & VFI & .12 & $8.92^{* *}$ & $1.20 \%$ \\
\hline & & & & VPA & .10 & $8.45^{* *}$ & $.80 \%$ \\
\hline & & & & ES & .04 & $3.33^{* *}$ & $.30 \%$ \\
\hline
\end{tabular}

Nota: VPA: Violencia del Profesorado hacia el Alumnado; VAPAE: Violencia del Alumnado hacia el Profesorado y Amenazas entre Estudiantes; VFA: Violencia Física entre el Alumnado; VFI: Violencia Física Indirecta; DA: Disrupción en el Aula; VAA: Violencia del Alumnado hacia el Alumnado. ES: Exclusión Social; ${ }^{* *}=p<.01$.

Asimismo, en la VFA presenta mayor poder de predicción la VAA $(\beta=.19 ; t=11.57$; $p<.01)$, seguida de la VAPAE $(\beta=.22 ; t=14.79 ; p<.01)$, la DA $(\beta=.21 ; t=15.25 ; p<.01)$, la VFI $(\beta=.12 ; t=8.92 ; p<.01)$, VPA $(\beta=.10 ; t=8.45 ; p<.01)$ y la $\mathrm{ES}(\beta=.04 ; t=3.33 ; p<.01)$. Aquí tres dimensiones (VAA, VAPAE, DA) explican el $46.7 \%$ de la varianza total de la violencia física del alumnado (VFA). La relación entre dimensiones es alta y positiva al obtener un valor de .70 en el coeficiente de correlación.

\section{Violencia indirecta ejercida por el alumnado}

Por un lado, en la violencia que ejerce el alumnado de forma indirecta (tabla 3), el resumen del modelo indica que en la violencia física indirecta (VFI) se han incluido todas las dimensiones (VAA, VFA, DE, VPA, VAPAE, ES). Dichas dimensiones explican un $40 \%$ de la varianza $(\mathrm{R}=.63 ; \mathrm{F}=549.963 \mathrm{p}<.01)$, con mayor peso de la VAA $(\beta=.27$; $t=14.90 ; p<.01)$, seguidas de la VFA $(\beta=.14 ; t=8.92 ; p<.01)$, la DA $(\beta=.15 ; t=10.08 ; p<.01)$ y la VPA $(\beta=.11 ; t=8.29 ; \beta<.01)$. Asimismo, con menor poder de predicción la VAPAE $(\beta=.08$; $t=5.14 ; p<.01)$ y ES ( $\beta=.05 ; t=3.12 ; \beta<.05)$. Por otro (tabla 3$)$, la disrupción en el aula (DA) es predicha por las dimensiones VAA $(\beta=.35 ; t=22.60 ; p<.01)$, VFA $(\beta=.21 ; t=15.23 ; p<.01)$, VFI $(\beta=.13 ; t=9.99 ; p<.01)$, ES $(\beta=.07 ; t=5.05 ; p<.01)$ y VPA $(\beta=.06 ; t=4.72 ; p<.01) ;$ quedando excluida la violencia del alumnado hacia el profesorado y amenazas entre estudiantes (VAPAE). Aquí, las tres dimensiones con mayor poder de predicción (VAA, VFA, VFI) explican el $44.90 \%$ de la varianza total en la disrupción escolar (DA), aunque asume el mayor peso factorial la violencia del alumnado hacia el alumnado (VAA $=39 \%)$. 
Tabla 3

Análisis de regresión lineal (pasos sucesivos), dimensiones criterio: VFI, DA y ES

\begin{tabular}{|c|c|c|c|c|c|c|c|}
\hline $\begin{array}{c}\text { Dimensión } \\
\text { criterio }\end{array}$ & $R$ & $R^{2}$ & $F$ & \multirow{2}{*}{$\begin{array}{c}\text { Dimensiones } \\
\text { Predictoras }\end{array}$} & \multirow[t]{2}{*}{$\beta$} & \multirow[t]{2}{*}{$t$} & \multirow[t]{2}{*}{$\begin{array}{l}\text { Varianza } \\
\text { Explicada }\end{array}$} \\
\hline \multirow[t]{7}{*}{ VFI } & .63 & .40 & $546.96(p<.001)$ & & & & \\
\hline & & & & VAA & .27 & $14.90^{* *}$ & $33 \%$ \\
\hline & & & & VFA & .14 & $8.92^{* *}$ & $3.40 \%$ \\
\hline & & & & DA & .15 & $10.08^{* *}$ & $1.65 \%$ \\
\hline & & & & VPA & .11 & $8.29 * *$ & $1.25 \%$ \\
\hline & & & & VAPAE & .08 & $5.14^{* *}$ & $.50 \%$ \\
\hline & & & & ES & .05 & $3.12^{*}$ & $.20 \%$ \\
\hline \multirow[t]{6}{*}{$\mathrm{DA}$} & .68 & .46 & $827.16(p<.001)$ & & & & \\
\hline & & & & VAA & .35 & $22.60^{* *}$ & $39 \%$ \\
\hline & & & & VFA & .21 & $15.23^{* *}$ & $4.50 \%$ \\
\hline & & & & VFI & .13 & $9.99^{* *}$ & $1.40 \%$ \\
\hline & & & & ES & .07 & $5.05^{* *}$ & $.60 \%$ \\
\hline & & & & VPA & .06 & $4.72^{* *}$ & $.50 \%$ \\
\hline \multirow[t]{6}{*}{ ES } & .62 & .39 & $626.98(p<.001)$ & & & & \\
\hline & & & & VAPAE & .31 & $19.39^{* *}$ & $32 \%$ \\
\hline & & & & VAA & .24 & $13.18^{* *}$ & $5.70 \%$ \\
\hline & & & & DA & .08 & $5.42^{* *}$ & $.80 \%$ \\
\hline & & & & VFA & .05 & $3.51^{* *}$ & $.30 \%$ \\
\hline & & & & VFI & .04 & $3.28^{* *}$ & $.20 \%$ \\
\hline
\end{tabular}

Nota: VPA: Violencia del Profesorado hacia el Alumnado; VAPAE: Violencia del Alumnado hacia el Profesorado y Amenazas entre Estudiantes; VFA: Violencia Física entre el Alumnado; VFI: Violencia Física Indirecta; DA: Disrupción en el Aula; VAA: Violencia del Alumnado hacia el Alumnado. ES: Exclusión Social; ${ }^{*}=p<.05 ;{ }^{* *}=p<.01$.

Finalmente, en cuanto a la predicción de la exclusión social (ES), los resultados muestran (tabla 3), una vez más, que las dimensiones con mayor poder de predicción son la VAPAE $(\beta=.31 ; t=19.39 ; p<.01)$ y la VAA $(\beta=.24 ; t=13.18 ; p<.01)$, y en un nivel mucho menor están la DA $(\beta=.08 ; t=5.42 ; \beta<.01)$, la VFA $(\beta=.05 ; t=3051 ; \beta<.01)$ y VFI $(\beta=.04$; $t=3.28 ; p<.01$ ). Estas dos dimensiones (VAPAE, VAA) explican el $37.7 \%$ de la varianza total de la ES, siendo significativa pero escasa (1.3\%) la aportación de las tres dimensiones restantes (DA, VFA, VFI). Destacar aquí, la exclusión de la dimensión violencia del profesorado hacia el alumnado (VPA). Los coeficientes de correlación (VFI: .63, DA: .68 y ES: .62) señalan una relación positiva y considerable entre las dimensiones.

\section{Discusión y conclusiones}

En términos generales, se puede afirmar que el grupo de dimensiones recogidas en el constructo violencia escolar, han obtenido una capacidad predictiva satisfactoria. Dentro de esta tendencia, la violencia del alumnado hacia el alumnado, la violencia física del alumnado y la violencia física indirecta están incluidas en el modelo pre- 
dictivo de todas las dimensiones, mientras que la violencia del profesorado hacia el alumnado no es predictiva de la exclusión social, la violencia del alumnado hacia el profesorado y amenazas entre estudiantes no es predictiva de la disrupción en el aula, la disrupción en el aula no es una dimensión predictiva de la violencia del alumnado hacia el profesorado y amenazas entre estudiantes, y finalmente, la exclusión social no es una dimensión predictiva de la violencia del profesorado hacia el alumnado.

Al analizar los resultados de la violencia del profesorado hacia el alumnado, su poder de predicción es bajo (todas las dimensiones que entran a formar parte explican un $30 \%$ de su varianza total). De igual forma, presenta un porcentaje medio de la varianza explicada muy reducido $(.84 \%)$ como dimensión predictora de las restantes dimensiones que conforman el constructo de la violencia escolar, no entrando a formar parte en la exclusión social. En este caso, los resultados pueden deberse a que la violencia que ejerce el profesorado hacia el alumnado es menos notoria en los centros educativos, aunque pueda llegar a acarrear consecuencias de mayor gravedad (Cabezas, 2008; Marchesi, Martín, Pérez \& Díaz, 2006).

Sin embargo, las evidencias obtenidas en la violencia del alumnado hacia el profesorado y amenazas entre estudiantes muestran a esta dimensión como un buen predictor de la violencia escolar (todas las dimensiones que entran a formar parte explican un $57 \%$ de su varianza total), siendo excluida de su predicción la disrupción en el aula. Además, presenta como dimensión predictora de las restantes dimensiones un porcentaje medio de varianza explicada aceptable (21.1\%), quedando excluida de la disrupción en el aula. Ello es congruente con la investigación del Defensor del Pueblo-UNICEF (2007), al sostener que aunque este tipo de violencia es poco frecuente, se ha agudizado lo suficiente para convertirse en un problema considerable en el sistema educativo.

En la misma línea, se constata a la violencia del alumnado hacia el alumnado como una buena predictora de la violencia escolar (las dimensiones implicadas explican un $64 \%$ de su varianza total) entrando a formar parte de todas las dimensiones. Presenta también, un porcentaje medio de varianza explicada aceptable (27.8\%) como dimensión predictora de las otras dimensiones. Estudios revisados (Coleman \& Hendry, 2003; Díaz-Aguado, Martínez \& Martín, 2004; Lisboa \& Koller, 2009; Oliva, Antolín, Estévez \& Pascual, 2012; Piñeiro, Arense \& Cerezo, 2013) sostienen que la reciprocidad en las relaciones, los procesos de interacción social, la cohesión, el contexto familiar, el apoyo y la amistad con los compañeros, constituyen un fuerte predictor de la conflictividad.

En el caso de la violencia física del alumnado, se confirma como una dimensión predictora de la violencia escolar aceptable (las dimensiones que engloba explican un $49 \%$ de su varianza total). Además, alcanza un porcentaje medio de varianza explicada por las restantes dimensiones bajo (2.84\%). Ello puede ser debido a que existe una relación inversa entre la gravedad de la conducta violenta y su incidencia, es decir, las conductas más graves son las de menor frecuencia (Navarro, Blanco \& Yubero, 2007). En consecuencia, la violencia física tiene menor presencia en los centros educativos (Caurcel, 2009; Defensor del Menor, 2006; Smith, 2005) lo cual conlleva un peor valor de predicción.

La violencia física indirecta exhibe un nivel de predicción regular (todas las dimensiones que entran a formar parte explican un $40 \%$ de su varianza total) constituyendo parte del conjunto de dimensiones. Asimismo, logra un porcentaje medio de varianza 
explicada por las restantes dimensiones bajo (1.42\%). Aquí, los resultados son dispares con otras investigaciones que señalan a este tipo de violencia como el más extendido en la etapa de educación secundaria obligatoria (Felip, 2007; Kunstche \& Klingemann, 2004), lo que debería convertirla en un buen predictor de violencia escolar.

Del mismo modo, la disrupción en el aula ofrece una predicción aceptable (las dimensiones aglutinadas explican un $46 \%$ de la varianza total) entrando a formar parte en todas las restantes dimensiones excepto en la violencia del alumnado hacia el profesorado y amenazas entre estudiantes. No obstante, como dimensión predictora de las dimensiones en las que forma parte, alcanza un porcentaje medio de la varianza explicada bajo (3.67\%). Los estudios realizados sobre comportamientos disruptivos contradicen este escaso valor de predicción, al ser considerada la más asidua en los centros de enseñanza (Badía, 2005; Muñoz, Carreras \& Braza, 2004).

Finalmente, la exclusión social presenta una baja predicción (todas las dimensiones que la conforman explican un $39 \%$ de la varianza total) entrando a formar parte de todas las dimensiones restantes excepto de la violencia del profesorado hacia el alumnado. Dentro de esta tendencia, ofrece porcentajes medios de varianza explicada como dimensión predictora en las restantes dimensiones baja (1.56\%). De nuevo, los resultados alcanzados son contradictorios con otras investigaciones que sostienen a la exclusión como un predictor relevante en la victimización de los adolescentes (Lisboa \& Koller, 2009).

La actual falta de coherencia entre la normativa o protocolos diseñados y los comportamientos o conductas habituales en los centros educativos es un claro indicador de la forma en que se aborda la prevención e intervención de la violencia escolar. En la mayoría de los centros de educación secundaria se han puesto en marcha planes y medidas a favor de la no violencia, pero se han ido acumulando sin estrategias de planificación coherentes que generen relaciones positivas en un clima colaborativo (Medina \& Cacheiro, 2010). Por lo tanto, parece evidente seguir trabajando en esta línea de investigación, de manera que permita a las comunidades educativas extraer conclusiones que les lleven a plantearse cómo facilitar su correcta puesta en marcha y qué medidas han de tomarse para conseguir un único objetivo: centros libres de violencia.

\section{Referencias}

Abramovay, M. (2005). Victimización en las escuelas. Ambiente escolar, robos y agresiones físicas. Revista Mexicana de Investigación Educativa. COMIE, 10(26), 833-864.

Álvarez-García, D., Álvarez, L., Núñez, J.C., González-Pienda J.A., Rodríguez, C., \& González-Castro, P. (2008). Estudio del nivel de violencia escolar en siete centros asturianos de Educación Secundaria. Aula Abierta, 36, 89-96.

Álvarez-García, D., Núñez, J.C., \& Dobarro, A. (2012). CUVE3. Cuestionario de Violencia Escolar - 3. Barakaldo: ALBOR-COHS.

Álvarez-García, D., Núñez, J.C., \& Dobarro, A. (2013). Cuestionarios para evaluar la violencia escolar en Educación Primaria y en Educación Secundaria: CUVE3-EP y CUVE3-ESO. Apuntes de Psicología, 31(2), 191-202. 
Arce, R., Seijo, D., Fariña, F., \& Mohamed-Mohand, L. (2010). Comportamiento antisocial en menores: Riesgo social y trayectoria natural de desarrollo. Revista Mexicana de Psicología, 27(2), 127-142.

Ato, M., López, J.J., \& Benavente, A. (2013). Un sistema de clasificación de los diseños de investigación en psicología. Anales de Psicología, 29(3), 1038-1059. Doi: 10.6018/ analesps.29.3.178511

Avilés, J.M. (2009): Victimización percibida y Bullying: Factores diferenciales entre víctimas. Boletín de Psicología, 95, 7-28.

Avilés, J.M., \& Monjas, I. (2005). Estudio de incidencia de la intimidación y el maltrato entre iguales en la educación secundaria obligatoria mediante el cuestionario CIMEI. Cuestionario sobre Intimidación y Maltrato Entre Iguales. Anales de psicología, 21(1), 27-41.

Badía, M.M. (2005). Gravedad e importancia de los comportamientos de indisciplina. Causas y factores asociados a ciertas conductas: una revisión de diferentes estudios. Psicología Educativa, 2(11), 65-78.

Benítez, J.L., \& Justicia, F. (2006). El maltrato entre iguales: Descripción y análisis del fenómeno. Electronic Journal of Research in Educational Psychology, 4, 81-93. Recuperado de http://www.investigacion-psicopedagogica.com/revista/new/ContadorArticulo. php?114

Cabezas, C. (2008). Violencia escolar: el acoso del profesor hacia el alumno. Madrid: CERSA.

Cajigas de Segredo, N., Kahan, E., Luzardo, M., Najson, S., Ugo, C., \& Zamalvide, G. (2006). Agresión entre pares (bullying) en un centro educativo de Montevideo: Estudio de las frecuencias de los estudiantes de mayor riesgo. Revista Médica del Uruguay, 22, 143-151.

Calvete, E., Orue, I., Estévez, A., Villardón, L., \& Padilla, P. (2010). Cyberbullying in adolescents: Modalities and aggressors' profile. Computers in Human Behavior, 26(5), 1128-1135. doi.org/10.1016/j.chb.2010.03.017

Caurcel, M.J. (2009). Estudio evolutivo del maltrato entre iguales desde la percepción y el razonamiento sociomoral de los implicados (Tesis Doctoral) Granada: Universidad de Granada.

Cava, M.J., Buelga, S., Musitu, G., \& Murgui, S. (2010). Violencia escolar entre adolescentes y en el ajuste psicosocial: un estudio longitudinal. Revista Psicopedagógica, 15(1), 21-34.

Cerezo, F. (2009). Analyzing Bullying in Spanish School. International Journal of Psychology and Psychological Therapy, 9(3), 383-394.

Coleman, J.C., \& Hendry, L.B. (2003). Psicología de la adolescencia. Madrid: Morata.

Cooley-Strickland, Q., Griffin, S. B., \& Furr-Holden, D. (2011). Efectos de la exposición de los adolescentes a la violencia en la comunidad: el Proyecto MORE. Psychosocial Intervention, 20, 131-148.

Corbetta, P. (2007). Metodología y Técnicas de Investigación Social (Primera edición). Madrid, España: McGraw-Hill.

Creswell, J.W. (2009). Research Design: Qualitative, Quantitative, and Mixed Methods Approaches. ( $3^{\underline{a}}$ ed.). Los Angeles: Sage Publications, Inc. 
Defensor del Menor de la Comunidad de Madrid (2006). Convivencia, conflictos y educación en los centros escolares de la Comunidad de Madrid. Madrid: Publicaciones del Defensor del Menor de la Comunidad de Madrid.

Defensor del Pueblo-UNICEF (2007). Violencia escolar: el maltrato entre iguales en la educación secundaria obligatoria. 1999-2006. Madrid: Publicaciones de la Oficina del Defensor del Pueblo. Recuperado de http://www.defensordelpueblo.es/es/Documentacion/ Publicaciones/monografico/Documentacion/Informe_violencia_escolar_ESO.pdf.

Del Moral, G., Suárez, C., \& Musitu, G. (2012). Roles de los iguales y bullying en la escuela: un estudio cualitativo. Revista de Psicología y Educación, 7, 105-127.

Díaz-Aguado, M. J. (2005). Porqué se produce la Violencia Escolar en las Escuelas y cómo prevenirla. Revista Iberoamericana de Educación, 37, 17-47.

Díaz-Aguado, M.J., Martínez, R., \& Martín, J. (2004). Prevención de la violencia y lucha contra la exclusión desde la adolescencia. Vol. 1. La violencia entre iguales en la escuela y en el ocio. Estudios comparativos e instrumentos de evaluación. Madrid: Instituto de la Juventud. Recuperado de http://www.injuve.es/sites/default/files/000-020-Violencia_indice.pdf.

Estévez, E. (2006). Violencia, victimización y rechazo escolar en la adolescencia. Valencia: Publicaciones de la Universidad de Valencia.

Felip, N. (2007). El acoso escolar. Revisión, análisis y contraste de algunas investigaciones. En J.J. Gálquez, M.C. Pérez, A.J. Cangas \& N. Yuste (Eds.). Situación actual y características de la violencia escolar (pp. 15-18). Almería: Grupo Editorial Universitario.

Gázquez, J.J., Pérez-Fuentes, M.C., Carrión, J.J., \& Santiuste, V. (2010). Estudio y análisis de conductas violentas en Educación Secundaria en España. Universitas Psychologica, 9(2), 371-380.

Gracia, E. \& Herrero, J. (2006). La comunidad como fuente de apoyo social: evaluación e implicaciones en los ámbitos individual y comunitario. Revista Latinoamericana de Psicología, 38, 327-342.

Guil, R., \& Mestre, J.M. (2004). Inteligencia emocional. En R. Guil (Coord.), Psicología Social para Psicopedagogos (pp. 165-212). Sevilla: Fénix Editora.

Kunstche, E., \& Klingemann, H. (2004). Hostility among Adolescents in Switzerland? Multivariate relations between excessive media use and forms of violence. Journal of Adolescents Health, 34, 230-236.

Lisboa, C., \& Koller, S. (2009). Factores protectores y de riesgo para la agresividad y victimización en escolares brasileños: el rol de los amigos. En C. Berger \& C. Lisboa (Eds.), Violencia Escolar. Estudios y posibilidades de intervención en Latinoamérica (1 ${ }^{a}$. Ed.) (pp. 161-181). Santiago de Chile: Universitaria.

Marchesi, A., Martín, E., Pérez, E.M., \& Díaz, T. (2006). Convivencia, conflictos y educación en los centros escolares de la Comunidad de Madrid. Madrid: Publicaciones del Defensor del Menor de la Comunidad de Madrid.

Martínez-Otero, V. (2005). Conflictividad escolar fomento de la convivencia. Revista Iberoamericana de Educación, 38, 33-52.

Martín-Seoane, G. (2008). La mediación como herramienta de prevención de la violencia escolar. Revista de Mediación, 1, 26-31.

Medina, A., \& Cacheiro, M.L. (2010). La prevención de la violencia: la implicación de la comunidad educativa para evitar situaciones de acoso escolar. Bordón, 62(1), 93-107. 
Muñoz, J.M., Carreras, M.R., \& Braza, P. (2004). Aproximación al estudio de las actitudes y estrategias de pensamiento social y su relación con los comportamientos disruptivos en el aula en la educación secundaria. Anales de Psicología, 20(1), 81-91.

Navarro, S., Blanco, A., \& Yubero, E. (2007). Convivir con la violencia: un análisis desde la psicología y la educación. La Mancha: Ediciones de la Universidad de Castilla.

Neuman, W.L. (2009). Social research methods: Quantitative and qualitative methods (7a Ed.). Boston, MA, EE. UU: Allyn \& Bacon.

Oliva, A., Antolín, L., Estévez, R., \& Pascual, D. (2012). Activos del barrio y ajuste adolescente. Psychosocial Intervention, 21, 17-27.

Ortega, R. (2010) (coord.). Agresividad injustificada, bullying y violencia escolar. Madrid: Alianza Editorial.

Ortega, R., \& Mora-Merchán, J.A. (2008). Las redes de iguales y el fenómeno del acoso escolar: Explorando el esquema dominio-sumisión. Infancia y Aprendizaje, 31(4), 515-528.

Palmero, F., Guerrero, C., Gómez, C., Carpi, A., \& Gorayeb, R. (2010). Manual de Teorías Emocionales y Motivacionales. Castellón: Universitat Jaume I.

Perren, S., Dooley, J.J., Shaw, T., \& Cross, D. (2010). Bullying in school and cyberspace: Associations with depressive symptoms in Swiss and Australian adolescents. Child and Adolescent Psychiatry and Mental Health, 4(28), 1-10.

Piñeiro, E., Arense, J.J., \& Cerezo, F. (2013). Contexto familiar y conductas de agresión y victimización entre escolares de educación secundaria. Bordón, 65(3), 109-129.

Rivera, M. (2011). Las voces en la adolescencia sobre bullying desde el escenario escolar. Estados Unidos de América: Palibrio.

Sánchez-Lacasa, C., \& Cerezo, F. (2010). Variables personales y sociales relacionadas con la dinámica bullying en escolares de Educación Primaria. Electronic Journal of Research in Educational Psychology, 8(3), 1015-1032.

Shapiro, J. (2006). Youth violence: the Problem and its Potential Solutions. Recuperado de:http://www.ucu.edu.uy/Facultades/Psicologia/TB/Documentos/Article_for_Uruguay.pdf

Smith, P.K. (2005). Violencia escolar y acoso: factores de riesgo familiares. En J. Sanmartín (Coord.), Violencia y Escuela (pp. 59-76). Valencia: Centro Reina Sofía.

Torrego, J.C. (2007). El modelo integrado: un nuevo marco educativo para la gestión de los conflictos de convivencia desde una perspectiva de Centro. Idea La ManchaRevista de Educación de Castilla-La Mancha, 4, 84-89.

Torrego, J.C., \& Moreno, J.M. (2003): Convivencia y Disciplina en la Escuela: El Aprendizaje de la Democracia. Madrid: Alianza.

Xunta de Galicia (2014). Datos e cifras do ensino non universitario. Santiago de Compostela: Consellería de Cultura, Educación e Ordenación Universitaria.

Fecha de recepción: 23 de mayo de 2016

Fecha de revisión: 23 de mayo de 2016

Fecha de aceptación: 02 de diciembre de 2016 


\section{ANEXO: Cuestionario}

\section{DATOS DEMOGRÁFICOS}

(Marca con una $\mathrm{X}$ la opción elegida en cada pregunta)

\begin{tabular}{|l|l|}
\hline 1. Edad: & 2. Género: Mujer. \\
\hline 3. ¿Qué estudios estás cursando?: \\
$\square 1^{\circ}$ de la ESO. $\square 2^{\circ}$ de la ESO. $\square 3^{\circ}$ de la ESO. $\square 4^{\circ}$ de la ESO.
\end{tabular}

Cuestionario CUVE3 - ESO

$\mathbf{1}=$ Nunca; $\mathbf{2}=$ Pocas veces; $\mathbf{3}=$ Algunas veces; $\mathbf{4}=$ Muchas veces; $\mathbf{5}=$ Siempre

\begin{tabular}{|c|c|c|c|c|c|}
\hline \multirow{3}{*}{ 1. El alumnado pone motes molestos a sus compañeros o compañeras. } & 1 & 2 & 3 & 4 & 5 \\
\hline & & & & & \\
\hline & & & & & \\
\hline $\begin{array}{l}\text { 3. Hay estudiantes que envían a compañeros/as mensajes a través del co- } \\
\text { rreo electrónico, de ofensa, insulto o amenaza. }\end{array}$ & & & & & \\
\hline 4. El alumnado habla con malos modales al profesorado. & & & & & \\
\hline 5. Ciertos estudiantes roban objetos o dinero del centro educativo. & & & & & \\
\hline $\begin{array}{l}\text { 6. Hay estudiantes que extienden rumores negativos acerca de compañe- } \\
\text { ros y compañeras. }\end{array}$ & & & & & \\
\hline $\begin{array}{l}\text { 7. Algunos estudiantes son discriminados por sus compañeros o compa- } \\
\text { ñeras por sus buenos resultados académicos. }\end{array}$ & & & & & \\
\hline $\begin{array}{l}\text { 8. Ciertos estudiantes roban objetos o dinero de otros compañeros o com- } \\
\text { pañeras. }\end{array}$ & & & & & \\
\hline 9. El profesorado tiene manía a algunos alumnos o alumnas. & & & & & \\
\hline $\begin{array}{l}\text { 10. Algunos estudiantes graban o hacen fotos a compañeros o compañe- } \\
\text { ras con el móvil, para burlarse. }\end{array}$ & & & & & \\
\hline 11. Los estudiantes hablan mal unos de otros. & & & & & \\
\hline $\begin{array}{l}\text { 12. Hay estudiantes que publican comentarios en Tuenti, Facebook de } \\
\text { ofensa, insulto o amenaza acerca de compañeros/as }\end{array}$ & & & & & \\
\hline $\begin{array}{l}\text { 13. Determinados estudiantes son discriminados por sus compañeros o } \\
\text { compañeras por sus bajas notas. }\end{array}$ & & & & & \\
\hline 14. El profesorado ridiculiza al alumnado. & & & & & \\
\hline $\begin{array}{l}\text { 15. Los estudiantes amenazan a otros de palabra para meterles miedo u } \\
\text { obligarles a hacer cosas. }\end{array}$ & & & & & \\
\hline 16. El alumnado falta al respeto a su profesorado en el aula. & & & & & \\
\hline $\begin{array}{l}\text { 17. El alumnado dificulta las explicaciones del profesor o profesora con su } \\
\text { comportamiento durante la clase. }\end{array}$ & & & & & \\
\hline 18. El profesorado ignora a ciertos alumnos o alumnas. & & & & & \\
\hline
\end{tabular}


19. Ciertos estudiantes envían a compañeros o compañeras mensajes con el móvil de ofensa, insulto o amenaza.

20. Los estudiantes insultan a profesores o profesoras.

21. El profesorado castiga injustamente.

22. Algunos estudiantes esconden pertenencias o material del profesorado, para molestarle deliberadamente.

23. Hay estudiantes que graban o hacen fotos a compañeros o compañeras con el móvil, para amenazarles o chantajearles.

24. El alumnado insulta a sus compañeros o compañeras.

25. El profesorado baja la nota a algún alumno o alumna como castigo.

26. Algunos estudiantes roban cosas del profesorado.

27. Hay estudiantes que graban o hacen fotos a profesores o profesoras con el móvil, para burlarse.

28. Determinados estudiantes pegan a compañeros o compañeras dentro del recinto escolar.

29. Ciertos profesores o profesoras intimidan o atemorizan a algún alumno o alumna.

30. Hay alumnado que ni trabaja ni deja trabajar al resto.

31. Determinados estudiantes causan desperfectos intencionadamente en pertenencias del profesorado.

32. Ciertos estudiantes publican comentarios en Tuenti, Facebook... de ofensa, insulto o amenaza a profesores o profesoras

33. Hay profesores y profesoras que insultan al alumnado.

34. Hay estudiantes que son discriminados por compañeros o compañeras por diferencias culturales, étnicas o religiosas.

35. Algunos estudiantes amenazan a otros/as con navajas $u$ otros objetos para meterles miedo u obligarles a hacer cosas.

36. Algunos estudiantes envían mensajes a compañeros/as a través del Tuenti, Facebook,.. de ofensa, insulto o amenaza.

37. El profesorado amenaza a algún alumno o alumna.

38. Algunos alumnos o alumnas protagonizan agresiones físicas en las cercanías del recinto escolar.

39. El profesorado no escucha a su alumnado.

40. El alumnado protagoniza peleas dentro del recinto escolar.

41. Los estudiantes publican en Internet fotos o vídeos ofensivos de compañeros o compañeras.

42. Algunos estudiantes son discriminados por sus compañeros o compañeras por su nacionalidad.

43. El alumnado dificulta las explicaciones del profesorado hablando durante la clase.

44. Los estudiantes publican en Internet fotos o vídeos ofensivos de profesores o profesoras.

\section{MUCHAS GRACIAS POR TU COLABORACIÓN}


